\title{
sciendo
}

\section{Design and fabrication of ${ }^{125}$ seeds for brachytherapy using capillary-based microfluidic technique}

\author{
Yuan Wang, \\ Miao Zhang, \\ Tong Song, \\ Zhenqi Chang
}

\begin{abstract}
A new kind of ${ }^{125}$ I seeds with a core-shell structure were synthesized by an easy assembling-disassembling coaxial capillaries microfluidic device. The dose distribution of a ${ }^{125}$ I brachytherapy source fabricated by arranging six ${ }^{125}$ I seeds collinearly within a cylindrical titanium capsule was simulated by modelling the source in a water phantom using Monte Carlo N-Particle Transport code. The influence of the motion and the core size of the ${ }^{125}$ I seeds on the dose distribution was also studied in this work.
\end{abstract}

Keywords: ${ }^{125}$ I seeds $\bullet$ Brachytherapy source $・$ Microfluidic $・$ Monte Carlo calculation $\bullet$ Core-shell

Y. Wang, M. Zhang, T. Song, Z. Chang ${ }^{\bowtie}$

University of Science and Technology of China

Department of Engineering and Applied Physics

Huang-Shan Road 443, He-Fei, P. R. China

E-mail: zqchang@ustc.edu.cn

Received: 1 March 2020

Accepted: 7 May 2020

\section{Introduction}

Iodine-125 is a widely used interstitial brachytherapy source employed in the treatment of cancer, especially in eye cancer and prostate cancer treatment [1]. Its radiation characteristics including the 27-32 keV X-ray and $35 \mathrm{keV} \gamma$-ray, with a comparatively long half-life (about 60 days) guarantee ${ }^{125} \mathrm{I}$ a recognized status in the radiation therapy modality through interstitial implantation [2]. Currently, the preparations of these implantation sources provided by commercial manufacturers mainly focus on the adsorption of ${ }^{125} \mathrm{I}$ on matrices made of palladium wires, ceramic beads, ion exchange resins, etc. [3-5]. However, due to the Intellectual Property Rights concerns and the commercial interests, little information about these techniques has been disclosed in the literature. In Majali et al. [6], an approach for the adsorption of ${ }^{125}$ I on palladium coated sliver wire sealed in titanium capsule as interstitial brachytherapy source was studied. And in the work of Song et al. [4], the optimized experimental conditions were given, which increased the adsorption efficiency of ${ }^{125}$ I up to $92 \%$. In recent years, Dash et al. [5] came up with a newly designed ${ }^{125} \mathrm{I}$ brachytherapy source, consisted of six ${ }^{125}$ I-absorbed palladium coated sliver spheres arranged collinearly within a cylindrical titanium capsule. Although the methods of ${ }^{125} \mathrm{I}$ adsorption can assist in the fabrication of a stable and effective ${ }^{125}$ I brachytherapy source, problems exist mainly in the fluctuation of the total dose and the dose distribution of ${ }^{125} \mathrm{I}$ of different design associated with the sensitivity of the absorption procedure. Therefore, the main aspect of our research effort is to find a more accurate and stable fabrication method. 
In this work, to fabricate a new category of ${ }^{125} I$ brachytherapy source with required properties such as the dosimetric accuracy and the dosage-uniformity, the ${ }^{125}$ I seeds with a core-shell structure were synthesized by a newly developed microfluidic device with two coaxial capillaries [7]. The core material is sodium iodine solution containing ${ }^{125} \mathrm{I}$ and the shell material is tri (propylene glycol) diacrylate (TPGDA) with photosensitizer. During microfluidic and core-shell structure droplets formation processes, the diameters of cores and shells can be precisely controlled at a range of $100-500 \mu \mathrm{m}$ and at $600 \mu \mathrm{m}$, respectively, by adjusting the flow rates [8]. During ultraviolet curing process, the shell phase of core-shell structure droplets is solidified by ultraviolet irradiation, which forms core-shell structure particles and that liquid cores containing ${ }^{125} \mathrm{I}$ are enclosed by solidified poly(tri (propylene glycol) diacrylate) (PTPGDA) shells (this particle hereinafter ${ }^{125}$ I seed or seed). Then, a couple of ${ }^{125} \mathrm{I}$ seeds are arranged collinearly within a cylindrical titanium capsule (hereinafter ${ }^{125} \mathrm{I}$ brachytherapy source). By this means, the dose of ${ }^{125} I$ in each ${ }^{125} I$ seed can be controlled precisely, and the nonuniform dose distribution as a result of the existence of dose gradient during the adsorption process could be eliminated. In addition, it is relatively convenient for physicians to alter the radiological dose by adding or reducing the number of ${ }^{125}$ I seeds, or by changing the volume of the core. Also, the efficiency of ${ }^{125} \mathrm{I}$ could be raised to nearly $100 \%$. Here, the dose distribution of this new ${ }^{125}$ I brachytherapy source was simulated by Monte Carlo modelling, and, the influences of the motion of ${ }^{125}$ I seeds motion within the titanium capsule and the size of core phase on the dose distribution were studied to a greater extent $[9,10]$.

\section{Materials and methods}

\section{Coaxial capillaries microfluidic device}

The experimental facilities includes capillaries with hydrophilic (fused silica tubing, Polymicro Technologies, INNOSEP Company, Zheng-Zhou, China) or hydrophobic (PEEK or PTFE tubings, Upchurch Scientific, INNOSEP Company, Zheng-Zhou, China) inner walls, gas-tight syringes, syringe pumps (PHD 2000, Harvard Apparatus), tubings (Polytetrafluoroethylene, Fisher Scientific Bioblock), T-junctions (P-728-01, Upchurch Scientific), line junction, and UV light (Lightning-cure LC8, Hamamatsu), as shown in Fig. 1. Two coaxial capillaries were inserted inside T-junction 1 along its main axis. The coaxial capillaries' tips exit the T-junction 2 at the center of an outlet PTFE tubing.

\section{Materials}

There are two types of disperse phase and one type of continuous phase which are mutually immiscible. Simethicone was used in continuous phase, and one of the disperse phase termed as the shell phase used
TPGDA (Aldrich), 96 wt\%: 1-hydroxycyclohexyl phenyl ketone (HCPK) as photoinitiator (Aldrich), $4 \mathrm{wt} \%$ [11]. The encapsulated phase used the core of the droplets consisted of sodium iodide solution $(8 \mathrm{wt} \%)$ containing radioactive ${ }^{125} \mathrm{I}(5 \mathrm{mCi})$. The mass densities of titanium, TPGDA, and dry air were $4.50,1.03$, and $1.20 \times 10^{-3} \mathrm{~g} / \mathrm{cm}^{3}$, respectively, and air composition by mass of nitrogen, oxygen, argon, and carbon, was $75.520,23.176,1.288$, and $0.016 \%$, respectively, were used for computational purposes [12]. Water consisted of two parts hydrogen, one part oxygen, with a mass density of $0.998 \mathrm{~g} / \mathrm{cm}^{3}$.

\section{Characterization}

An optical microscope (XSP-30E, Shanghai Halibut Instrument Limited Company, China) equipped with a charge-coupled device (CCD) camera (uEye, UI-2220SE, IDS) was used to observe the formation of the core-shell structure droplets directly. The camera captures up to $52 \mathrm{ftps}$ at a full resolution of 768 pixels $\times 576$ pixels. Then during the flowing in the tube, droplets were polymerized under the explosion of UV irradiation by means of an UV source operating at $\lambda=365 \mathrm{~nm}$ online. The overall diameter and the core diameter of the ${ }^{125}$ I seed were measured with an image analysis software (uEye Cockpit) operating the CCD camera. The dosimetry parameters of the new source were calculated by Version 5 of Monte Carlo N-Particle Transport (MCNP5) code. The results of the tally were fed to a plotting software, SURFER 11.0, used to construct the isodose curves against dose values calculated above.

\section{Results and discussion}

\section{Fabrication of ${ }^{125} \mid$ seeds for ${ }^{125} \mid$ brachytherapy source}

For the preparation of ${ }^{125} \mathrm{I}$ seeds, core-shell structure droplets are produced first. A flexible coaxial capillaries microfluidic device was designed by us to produce core-shell droplets consisted of liquid core surrounded by a layer of TPGDA. The main part or element of the liquid core was sodium iodide solution containing radioactive ${ }^{125} \mathrm{I}$. As shown in Fig. 2b, sodium iodide solution and TPGDA were delivered by the inner capillary and the outside capillary, respectively, with the inner one was fixed inside of the outside one. $Q_{1}, Q_{2}$, and $Q_{3}$ were the flow rates of continuous phase, shell phase, and core phase, respectively. The reaction between surface tension and shear force in the shell phase caused the formation of core droplets [13], at the tip of the inner capillary. Subsequently, shell phase generated at the end of the outside capillary due to the shear force of continuous phase happened between the outside capillary and the PTFE tubing. As a result, the core phase was encapsulated in the shell material, and the core-shell structure droplet was fabricated. Then, the TPGDA shell was polymerized downstream by photo-induced polym- 


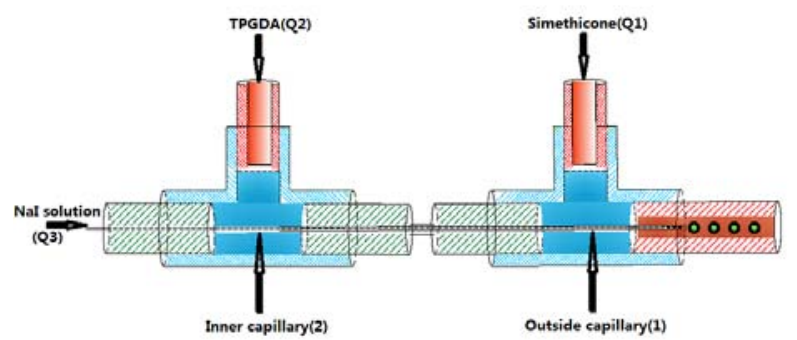

Fig. 1. Schematic drawing of capillary-based microfluidic device for synthesis of core-shell microspheres. Inner/ outer diameter of the outside capillary (1) is $255 \mu \mathrm{m} /$ $510 \mu \mathrm{m}$, and inner/outer diameter of the inner capillary (2) is $100 \mu \mathrm{m} / 167 \mu \mathrm{m}$.
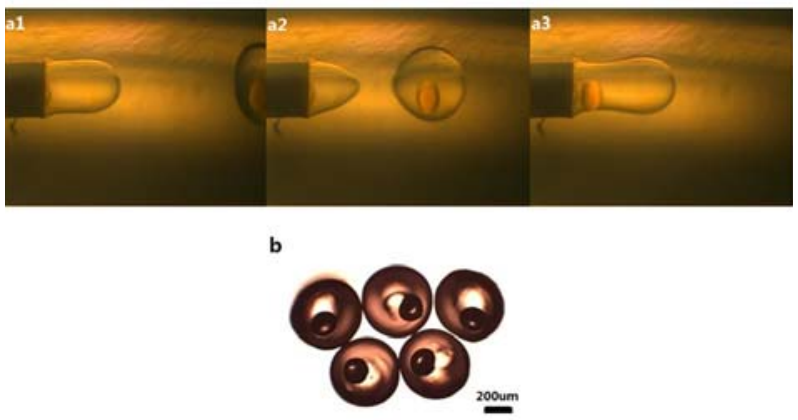

Fig. 2. (a1-a3) Formation of core-shell structure droplet, $Q_{1}=120 \mu \mathrm{L} / \mathrm{min}, Q_{2}=3.75 \mu \mathrm{L} / \mathrm{min}, Q_{3}=0.25 \mu \mathrm{L} / \mathrm{min}$; (b) optical image of as-prepared ${ }^{125}$ I seeds.

erization to form the seeds with ${ }^{125}$ I core-PTPGDA shell structure. Polyacrylate possesses an as-desired radiation resistance. Researches of poly(tert-butyl acrylate) (PTBA) radiation resistance indicated that the thickness of irradiation-damaged polyacrylate file was about $0.1 \AA$ after 1 minute irradiation under $9.659 \mathrm{keV}$ photons at a flux density of $0.4 \times 10^{9}$ photons $/\left(\mathrm{s} \cdot \mathrm{mm}^{2}\right)$ [14]. For a $0.5 \mathrm{mCi}$ as-prepared ${ }^{125} \mathrm{I}$ seed, it was estimated that the thickness of irradiation-damaged PTPGDA shell would be $<1 \mu \mathrm{m}$ after a year, which assured that TPGDA was a competent material for the shell of the ${ }^{125}$ I seed.

The droplet formation from two continuous flowing liquids was achieved through either dripping or jetting mode [15], and the key parameters for mastering the droplet formation mode are the flow rates of the continuous and dispersed phase. In this work, by adjusting the fluid flow rate, the droplets were formed in the dripping mode which ensured the formation of highly monodisperse core-shell structure droplets [16]. The formation of the core-shell structure droplet and the as-prepared ${ }^{125} \mathrm{I}$ seeds with uniform core size and overall seed size were shown in Fig. 2.

\section{Control of ${ }^{125} \mid$ contents in a ${ }^{125} \mid$ seed}

For the treatment of different tumour sites, a source with various radiation doses for brachytherapy is required. The dose delivered by as-prepared ${ }^{125} \mathrm{I}$ brachytherapy source is controlled by the dosage of ${ }^{125} \mathrm{I}$ in each ${ }^{125} \mathrm{I}$ seed and the number of the seeds sealed in the titanium capsule. Because of the limi- tation on the length of the source in practical utilization, controlling the content of ${ }^{125} \mathrm{I}$ in each seed precisely to meet the radiation dose requirements has to be approached realistically. The ${ }^{125}$ I content of each seed is related to the ${ }^{125} \mathrm{I}$ concentration in feed solution of the core and the core size of as-designed seed. By keeping size of the core droplet constant, it is easy to understand that the proportional increase of radiation dose rate of the seeds with the increase of the ${ }^{125} \mathrm{I}$ concentration in core feed solution. While keeping the concentration of ${ }^{125} \mathrm{I}$ in core feed solution fixed, the content of ${ }^{125} \mathrm{I}$ in each seed can be controlled by changing the size of core droplet, and increases as the core size increases. In our previous work, the control of overall core-shell structure's droplet size, core size, and shell size were studied in detail by adjusting the flow rates of different phase in the coaxial capillary microfluidic device and the relationship between core size and flow rate of the related fluids can be described by the formula:

$$
r_{\text {core }}=\sqrt[\varepsilon]{\frac{Q_{3}}{Q_{2}+Q_{3}}} \times r_{\text {seed }}
$$

where $r_{\text {core }}$ and $r_{\text {seed }}$, are the diameter of the core droplet and the seed, respectively, whereas $Q_{2}$ and $Q_{3}$ are the flow rate of the shell phase and the core phase, respectively. In the experiments, the precise control of core size can be achieved by adjusting flow rate of core and shell phase. Also, the size-designated overall core-shell structure droplet can also be obtained by changing the flow rate of core, shell, and continuous phase, respectively. The shell phase of the core-shell structure droplets were subsequently polymerized while flowing in the tubing under ultraviolet irradiation, and ${ }^{125}$ I seeds were fabricated finally. The as-prepared seeds with the same overall size but with different core sizes were shown in Fig. 3 and were of narrow size distribution of the cores and seeds, which were beneficial in keeping the same dose rate of the seeds. If a reduction in PTPGDA shell thickness by less than $10 \%$ is considered and still to ensure that the ${ }^{125} \mathrm{I}$-containing solution was not leaked during a half-life period of ${ }^{125} \mathrm{I}$ (about 60 days), a single ${ }^{125}$ I seed with the maximum radioactivity $49 \mathrm{mCi}$, with core diameter of $500 \mu \mathrm{m}$ and shell thickness of $50 \mu \mathrm{m}$, calculated based on the radiation resistance of acrylate was used.
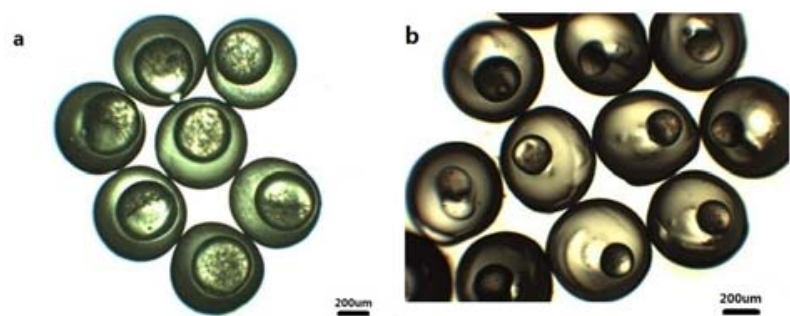

Fig. 3. Optical images of ${ }^{125} \mathrm{I}$ seeds with a various core diameter and the same overall diameter of $600 \mu \mathrm{m}$. (a) Core diameter of $400 \mu \mathrm{m}, Q_{1}=120 \mu \mathrm{L} / \mathrm{min}, Q_{2}=3 \mu \mathrm{L} / \mathrm{min}$, $Q_{3}=1 \mu \mathrm{L} / \mathrm{min}$, the radioactivity of a single ${ }^{125}$ I-encapsulated microsphere was about $0.17 \mathrm{mCi}$; (b) core diameter of $200 \mu \mathrm{m}, Q_{1}=120 \mu \mathrm{L} / \mathrm{min}, Q_{2}=3.83 \mu \mathrm{L} / \mathrm{min}, Q_{3}=$ $0.17 \mu \mathrm{L} / \mathrm{min}$, the radioactivity of a single ${ }^{125} \mathrm{I}$-encapsulated microsphere was about $0.02 \mathrm{mCi}$. 

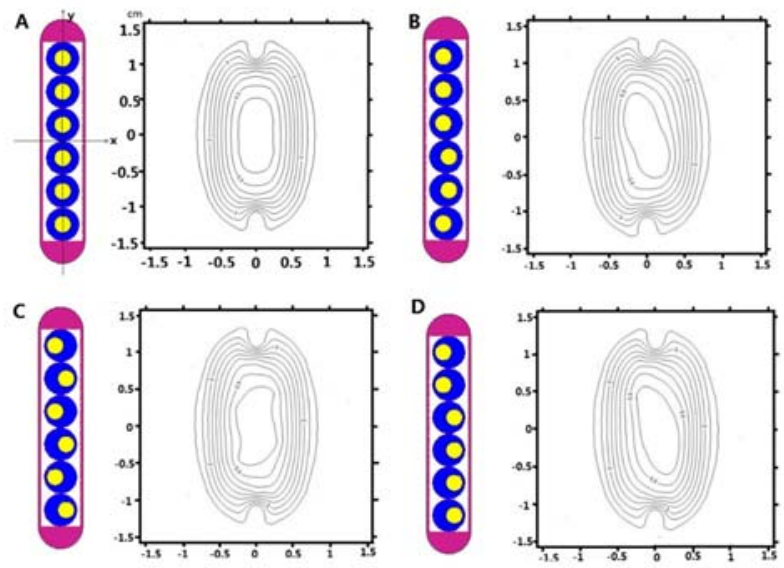

Fig. 4. Isodose curves of the ${ }^{125} \mathrm{I}$ brachytherapy sources with different orientation of cores in the sources. Overall diameter of the ${ }^{125} \mathrm{I}$ seeds was $600 \mu \mathrm{m}$, and core diameter was $300 \mu \mathrm{m}$. (a) Each seed of core and shell is of homocentricity. (b-d) Each seed of core and shell was of nonhomocentricity.

\section{Dose distribution of the new ${ }^{125} \mid$ brachytherapy source}

A structured source with a titanium capsule size of $800 \mu \mathrm{m}$ outer diameter, $700 \mu \mathrm{m}$ inner diameter, and $4 \mathrm{~mm}$ length was used in clinical medicine conventionally and the capsules filled with six as-prepared seeds of $600 \mu \mathrm{m}$ diameter (shown in Fig. 4) were used for an evaluation of its dose distribution, and the dosimetry parameters of the source were calculated by MCNP5 code [17]. The simulation considered a spherical water phantom and the dose values in water were calculated at radial distances of 0-2 cm. The DLC-189 cross section was used for transport calculations and the Integrated TIGER Series (ITS) three-dimensional (3D) code system was used for coupled photon-electron transport. In order to determine relevant dose distribution accurately, different size of voxel computational cells were placed in select positions. The total number of photons transported per calculation run was $10^{9}$ with statistical uncertainties below 3.6\%. A plotting software, SURFER 11.0 was used to construct the isodose curves.

If the core and shell of each seed have concentricity, a ${ }^{125}$ I brachytherapy source containing six seeds that lined the capsule appears to have an ellipsoid-like uniform dose distribution, as shown in Fig. 4a. In fact, it is difficult to synthesize a homocentric seed in the microfluidic system since the core droplet is free to move in the double droplet before polymerization of the seed shell, which will induce nonuniform distribution of the dose.

The core center usually deviates from the seed center in microfluidic fabrication of the seed with a core-shell structure. The maximum deviation of the core center from shell center, in that the core tangent to surface of the seed, was modelled for exploring the influence of the core position on dose distribution of the source. The position of six cores was described by setting $x, y$ coordinates in $x y$ plane. Obviously,
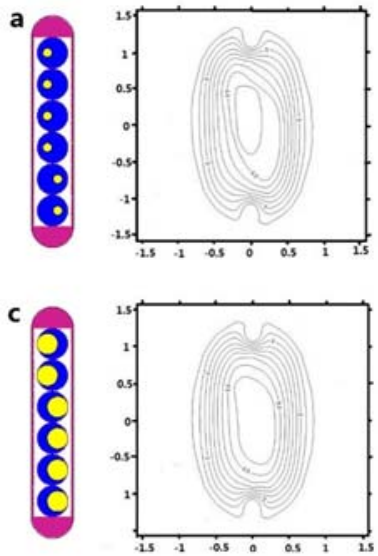
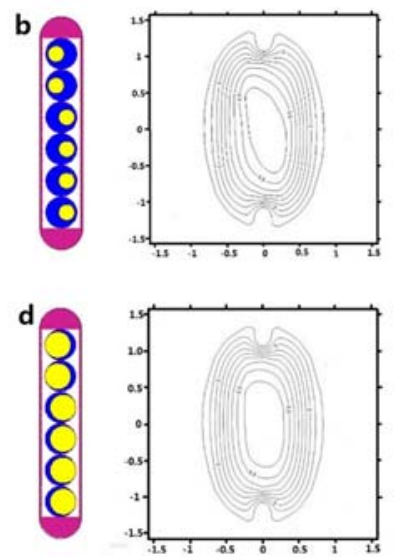

Fig. 5. Isodose curves of the ${ }^{125}$ I brachytherapy sources with different core size under the same orientation in the source. Each seed diameter was $600 \mu \mathrm{m}$, and core diameter was (a) $150 \mu \mathrm{m}$, (b) $300 \mu \mathrm{m}$, (c) $400 \mu \mathrm{m}$, and (d) $500 \mu \mathrm{m}$.

the more deviation of the core center from the seed center is, the less uniform dose distribution of the source exists. For a size- and position-confined seed in the capsule, the increase in the core size decreases the deviation of core center from its ideal center and more uniform dose distribution of the ${ }^{125}$ I brachytherapy source was produced. The model of the core orientation in the source was randomly selected, as shown in Fig. 4b-d, to illustrate the influences of the core position on the dose distribution of the ${ }^{125} I$ brachytherapy source.

The isodose curves of cores with diameter of 150 , 300,400 , and $500 \mu \mathrm{m}$ constructed by SURFER 11.0 were shown in Fig. 5, respectively, and found that when the diameter of core was $150 \mu \mathrm{m}$, the dose distribution had a less uniformity within $\pm 5 \mathrm{~mm}$ in $x$ axis, but beyond $\pm 5 \mathrm{~mm}$, the dose distribution was uniform. When core size was increased from $150 \mu \mathrm{m}$ to $400 \mu \mathrm{m}$, the uniform dose distribution within $\pm 5 \mathrm{~mm}$ was improved, but was still nonuniform. But with core size $500 \mu \mathrm{m}$, the dose distribution of the ${ }^{125} \mathrm{I}$ brachytherapy source was close to the ideal model.

By keeping the core diameter of $500 \mu \mathrm{m}$, the dose distribution of other modelled sources with a different orientation of the cores was also computed, as shown in Fig. 6. The results indicated that the dose distribution of the ${ }^{125}$ I brachytherapy sources with various orientations of the cores exhibited negligible differences or variations and so we can conclude that all of them were uniform even within $\pm 5 \mathrm{~mm}$ in $x$ axis and $y$ axis. Therefore, the ${ }^{125}$ I seeds with a
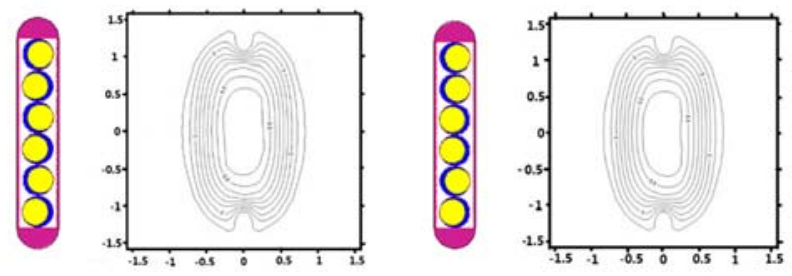

Fig. 6. Isodose curves of the ${ }^{125} \mathrm{I}$ brachytherapy sources with a different orientation of the cores. Overall diameter of the ${ }^{125}$ I seeds was $600 \mu \mathrm{m}$, and the diameter of core was $500 \mu \mathrm{m}$. 


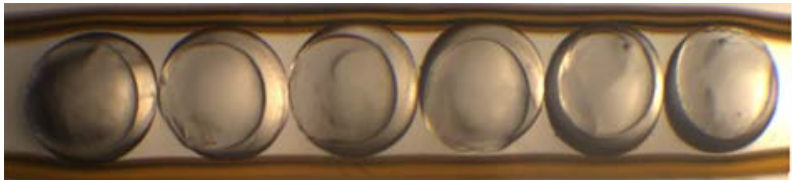

Fig. 7. Optical image of a ${ }^{125} \mathrm{I}$ brachytherapy source consisted of six ${ }^{125}$ I seeds within a glass tubing. The overall diameter of the seeds was $600 \mu \mathrm{m}$, and the diameter of core was $500 \mu \mathrm{m}$.

core size of $500 \mu \mathrm{m}$ and seed size of $600 \mu \mathrm{m}$ were designed and fabricated. Additionally, an inactive dummy ${ }^{125}$ I brachytherapy source was fabricated by encapsulating six as-prepared ${ }^{125} \mathrm{I}$ seeds in a glass tube with the size of $600 \mu \mathrm{m} / 850 \mu \mathrm{m}$ inside/outside diameter (ID/OD), as shown in Fig. 7.

\section{Conclusions}

A new ${ }^{125}$ I seeds with core-shell structure for ${ }^{125} \mathrm{I}$ brachytherapy source was fabricated in a capillary-based microfluidic device. In comparison with the conventional adsorption methods for the fabrication of ${ }^{125}$ I seed, an encapsulating approach provided a precise load of the quantity-demanded ${ }^{125} \mathrm{I}$ in a seed with a close to $100 \%$ of encapsulation efficiency. The ${ }^{125} \mathrm{I}$ seed and its core size can be controlled by simply adjusting the flow rates of the fluids in the microfluidic system. The dose distribution of the ${ }^{125} \mathrm{I}$ brachytherapy source consisted of the as-prepared seeds and titanium capsule was calculated by MCNP5, and the results showed that the nonuniformity of the dose distribution induced by the deviation of the core from the center of the whole seed could be eliminated by increasing the core size for a size-prescribed capsule.

Acknowledgments. This work was financially supported by the National Key R\&D Program of China (grant no. 2017YFE0300504) and the National Nature Science Foundation of China (grant no. 21978279). Also, the authors thank the Key Research and Development Projects in Anhui Province of the People's Republic of China (no. 201904a05020048) for financial support in the research.

\section{References}

1. Zhang, F. J., Wu, P. H., Zhao, M., Huang, J. H., Fan, W. J., Gu, Y. K., Liu, J., Zhang, L., \& Lu, M. J. (2006). CT guided radioactive seed ${ }^{125} \mathrm{I}$ implantation in treatment of pancreatic cancer. Natl. Med. J. Chin., 86(4), 223-227. (in Chinese).

2. Chino, K., Silvain, D., Grace, A., Stubbs, J., \& Stea, B. (2008). Feasibility and safety of outpatient brachytherapy in 37 patients with brain tumors using the GliaSite (R) Radiation Therapy System. Med. Phys., 35(7), 3383-3388. https://doi. org/10.1118/1.2940602.

3. Popescu, C. C., Wise, J., Sowards, K., Meigooni, A. S., \& Ibbott, G. S. (2000). Dosimetric charac- teristics of the Pharma Seed (TM) model BT-125-I source. Med. Phys., 27(9), 2174-2181. https://doi. org/10.1118/1.1289897.

4. He, J., Song, H., Jian, Y., Jiang, L., Zhong, W., Li, X., Ma, Z., \& Liu, G. (2011). Adsorption of I-125 on palladium coated silver wire. J. Radioanal. Nucl. Chem., 290(2), 469-473. https://doi.org/10.1007/ s10967-011-1334-1.

5. Saxena, S. K., Sharma, S. D., Dash, A., \& Venkatesh, M. (2009). Development of a new design I-125-brachytherapy seed for its application in the treatment of eye and prostate cancer. Appl. Radiat. Isot., 67(7/8), 1421-1425. https://doi.org/10.1016/j. apradiso.2009.02.040.

6. Mathew, C., Majali, M. A., \& Balakrishnan, S. A. (2002). A novel approach for the adsorption of iodine-125 on silver wire as matrix for brachytherapy source for the treatment of eye and prostate cancer. Appl. Radiat. Isot., 57(3), 359-367. https://doi. org/10.1016/S0969-8043(02)00099-4.

7. Steinbacher, J. L., \& McQuade, D. T. (2006). Polymer chemistry in flow: New polymers, beads, capsules, and fibers. J. Polym. Sci. Pol. Chem., 44(22), 6505-6533. https://doi.org/10.1002/pola.21630.

8. Chang, Z., Serra, C. A., Bouquey, M., Prat, L., \& Hadziioannou, G. (2009). Co-axial capillaries microfluidic device for synthesizing size- and morphologycontrolled polymer core-polymer shell particles. $L a b$ Chip, 9(20), 3007-3011. https://doi.org/10.1039/ b913703c.

9. Rivard, M. J. (2001). Monte Carlo calculations of AAPM Task Group Report No. 43 dosimetry parameters for the MED3631-A/(MI)-I-125 source. Med. Phys., 28(4), 629-637. https://doi.org/10.1118/1.1355306.

10. Rivard, M. J., Coursey, B. M., DeWerd, L. A., Hanson, W. F., Huq, M. S., Ibbott, G. S., Mitch, M. G., Nath, R., \& Williamson, J. F. (2004). Update of AAPM Task Group No. 43 Report: A revised AAPM protocol for brachytherapy dose calculations. Med. Phys., 31(3), 633-674. https://doi.org/10.1118/1.1646040.

11. Chang, Z., Serra, C. A., Bouquey, M., Kraus, I., Li, S., \& Koehler, J. M. (2010). Multiscale materials from microcontinuous-flow synthesis: $\mathrm{ZnO}$ and $\mathrm{Au}$ nanoparticle-filled uniform and homogeneous polymer microbeads. Nanotechnology, 21(1), 015605. https://doi.org/10.1088/0957-4484/21/1/015605.

12. Weast, C. R. (Ed.).(1984). CRC Handbook of chemistry and physics (65 ed.) Boca Raton: Chemical Rubber Company.

13. Serra, C. A., \& Chang, Z. (2008). Microfluidicassisted synthesis of polymer particles. Chem. Eng. Technol., 31(8), 1099-1115. https://doi.org/10.1002/ ceat. 200800219.

14. Richter, A. G., Guico, R., Shull, K., \& Wang, J. (2006). Thickness and interfacial roughness changes in polymer thin films during X-irradiation. Macromolecules, 39(4), 1545-1553. https://doi.org/10.1021/ma050060v.

15. Utada, A. S., Chu, L. -Y., Fernandez-Nieves, A., Link, D. R., Holtze, C., \& Weitz, D. A. (2007). Dripping, jetting, drops, and wetting: The magic of microfluidics. MRS Bull., 32(9), 702-708. https://doi.org/10.1557/ mrs2007.145.

16. Utada, A. S., Lorenceau, E., Link, D. R., Kaplan, P. D., Stone, H. A., \& Weitz, D. A. (2005). Monodisperse 
double emulsions generated from a microcapillary device. Science, 308(5721), 537-541. https://doi. org/10.1126/science.1109164.
17. X-5 Monte Carlo Team. (2005). MCNP - A General Monte Carlo N-particle Transport Code, Version 5. Los Alamos: Los Alamos National Laboratory. 